\title{
Desenvolvimento larval de Brycon hilarii (Characiformes, Characidae)
}

\author{
Fernando G. de Oliveira, Andréa Bialetzki, Luiz C. Gomes, Miriam Santin \& Tátia L. Taguti
}

Laboratório de Ictioplâncton, Núcleo de Pesquisas em Limnologia, Ictiologia e Aquicultura e Programa de Pós-Graduação em Ecologia de Ambientes Aquáticos Continentais, Departamento de Biologia, Universidade Estadual de Maringá (UEM). Av. Colombo, 5790, 87020-900, Maringá, Paraná, Brasil. (fgoliveira.bio@gmail.com, bialetzki@nupelia.uem.br)

\begin{abstract}
Larval development of Brycon hilarii (Characiformes, Characidae). The present study characterizes the early stages of development of Brycon hilarii (Valenciennes, 1850) based on morphometric and meristic characters. Patterns of development in morphometric characters were analyzed through the fit of linear, quadratic and linear piecewise models. Samples were collected from March 2000 to March 2004 in basins of the Cuiabá and Manso rivers, state of Mato Grosso, Brazil. Larvae were identified and classified in different stages (Larval Yolk, Pre-Flexion, Flexion, and Post-Flexion) according to notochord development. The morphometric and meristic characters for each larva were, respectively, measured and counted. The standard length ranged from 3.25 to $26.00 \mathrm{~mm}$. The Larval Yolk stage showed a greater concentration of dendritic pigments along the intestine, and the Flexion and Post-Flexion stages showed an intensified pigmentation intensified along the back. A single spot on the humerus and another on the caudal peduncle were observed at the end of the Post-Flexion stage. Also, in the Larval Yolk and Pre-Flexion stages. The intestine is long, the snout is short, the adhesive organ was present in the Larval Yolk and Pre-Flexion, and the mouth is terminal throughout the development. The total number of myomeres ranged from 41 to 50 ( 23 to 30 pre-anal and 16 to 24 post-anal). Number of fin rays: dorsal 11; anal 30; pectoral 15 and ventral eight. The analysis of the development indicated higher metamorphosis in the Flexion stage.
\end{abstract}

KEYWORDS. Morphology, larvae, fish, piraputanga, Paraguai river basin.

RESUMO. O presente estudo caracterizou o desenvolvimento inicial de Brycon hilarii (Valenciennes, 1850) através de caracteres morfométricos e merísticos e analisou as relações entre as características morfométricas das larvas ao longo do desenvolvimento usando modelos de regressão linear, quadrática e linear por partes. O material foi obtido nas bacias dos rios Cuiabá e Manso, Mato Grosso, Brasil, entre março de 2000 e março de 2004. Os indivíduos foram identificados e separados de acordo com o grau de desenvolvimento da notocorda e, posteriormente, medidos e contados os caracteres morfométricos e merísticos, respectivamente. O comprimento padrão variou entre 3,25 e $26,00 \mathrm{~mm}$. Inicialmente, as larvas apresentaram maior concentração de pigmentos dendríticos ao longo do intestino e, em flexão e pós-flexão, intensificaram-se no dorso do corpo. Uma mancha umeral e outra no pedúnculo caudal foram observadas no final do estágio de pós-flexão. O intestino apresentou-se longo, o focinho curto, o órgão adesivo presente em larval vitelino e pré-flexão e a boca terminal por todo o desenvolvimento. O número total de miômeros variou de 41 a 50 ( 23 a 30 pré e 16 a 24 pós-anal). O número de raios das nadadeiras foi: dorsal 11; anal 30; peitoral 15 e ventral oito. A análise do crescimento indicou maior metamorfose no estágio de flexão.

PALAVRAS-CHAVE. Morfologia, larva, peixe, piraputanga, bacia do rio Paraguai.

O conhecimento acerca do desenvolvimento inicial dos peixes é essencial para a correta identificação das larvas em seu ambiente. Entretanto, estudos taxonômicos das larvas são realizados em um cenário de grandes dificuldades de identificação e escassa literatura comparativa. Morfologicamente, as larvas de peixes são muito diferentes dos adultos e, em alguns casos, são consideradas como novos gêneros e até classificadas em famílias distintas das quais realmente pertencem (Leis \& TRNSKI, 1989). Desta forma, a caracterização do desenvolvimento inicial dos peixes é de fundamental importância para estudos taxonômicos auxiliando o entendimento das relações ecológicas das fases iniciais do ciclo de vida das espécies com o ambiente em que vivem.

O gênero Brycon Müller \& Troschel 1844 (Bryconinae, Characidae) compreende cerca de 40 espécies válidas (HowES, 1982; BRITSKI et al., 1988; Ceccarelli \& Senhorini, 1996; Lima, 2003). Dentro da ordem Characiformes, este gênero se destaca pela presença de algumas espécies com elevado interesse na pesca e grande potencial zootécnico, como o matrinxã (Brycon amazonicus (Spix \& Agassiz, 1829)), a piracanjuba (B. orbignyanus (Valenciennes, 1850)), a piabanha (B. insignis Steindachner, 1877) e a piraputanga (B. hilarii (Valenciennes, 1850)) (LiMA, 2003).

Brycon hilarii é uma espécie reofílica e pode ser encontrada em locais de remansos, embaixo de árvores frutíferas e próxima a plantas aquáticas. Esta espécie tem um amplo espectro alimentar (REYs et al., 2009) e é uma importante dispersora de sementes sendo uma das principais responsáveis, principalmente no período de migração reprodutiva, pela distribuição das espécies vegetais ao longo da mata ciliar (BANACK et al., 2002; REYS et al., 2009). Seu comprimento total pode chegar a $56 \mathrm{~cm}$ (Froese \& Pauly, 2011) e a sua distribuição é registrada na bacia do rio Paraguai, América do Sul (Paraguai e Brasil) (LimA, 2003). Recentemente, indivíduos dessa espécie foram introduzidos na planície de inundação do alto rio Paraná tendo sua presença registrada por GraÇA \& PAVANELli (2007).

Dentre as espécies de Brycon, B. cephalus (Günther, 1869) (Lopes et al., 1995; Romagosa et al., 2001), B. insignis (Andrade-Talmelli et al., 2001), B. guatemalensis (Regan, 1908) (ARIAS, 2006), B. orbignyanus (NAKATANi et al., 2001; Reynalte-TATAJE et al., 2004; Correia et al., 2010), B. gouldingi Lima, 2004 (FAustino et al., 2010) e B. orthotaenia Günther, 1864 (Gomes et al., 2011) foram descritas considerando pelo menos parte do seu desenvolvimento inicial, porém, para B. hilarii, esse estudo é inexistente. Assim, considerando a relevância ecológica, pesqueira e zootécnica desta espécie e a importância de caracterizar e reconhecer suas fases larvais, este estudo tem como objetivos (i) descrever morfologicamente os estágios larvais de $B$. hilarii utilizando caracteres morfométricos 
e merísticos e (ii) verificar possíveis mudanças de taxas de crescimento ao longo do desenvolvimento dos estágios larvais por meio da análise das relações entre caracteres morfológicos, utilizando modelos analíticos de regressão.

\section{MATERIAL E MÉTODOS}

Área de estudo. O material utilizado neste estudo foi obtido em amostragens realizadas em 18 estações localizadas nas bacias dos rios Cuiabá e Manso $\left(14^{\circ} 41,88^{\prime} \mathrm{S}, 56^{\circ} 15,27^{\prime} \mathrm{W}\right.$ e $\left.15^{\circ} 58,44^{\prime} \mathrm{S}, 55^{\circ} 56,44^{\prime} \mathrm{W}\right)$ (Fig. 1).

Coleta das larvas. As coletas foram realizadas no período de março de 2000 a março de 2004, com redes de plâncton do tipo cônico-cilíndricas de malha $0,5 \mathrm{~mm}$ (conforme NAKATANi et al., 2001). Em cada estação de amostragem foram realizadas coletas de superfície, sendo uma diurna $(16: 00 \mathrm{~h})$ e outra noturna (22:00 h), com exceção das estações 11 e 12, onde as coletas foram realizadas ao longo de um ciclo nictemeral com intervalo de quatro horas entre as amostragens. Em cada estação de coleta foi realizada também uma amostragem não padronizada na região marginal utilizando-se peneirão (NAKATANi et al., 2001), com o objetivo de capturar larvas associadas às macrófitas aquáticas. As amostras coletadas foram acondicionadas em frascos de polietileno e fixadas em formol $4 \%$ tamponado com carbonato de cálcio.

Caracterização dos estágios de desenvolvimento. Em laboratório as amostras foram triadas, as larvas separadas e, em seguida, identificadas através da técnica de sequência regressiva de desenvolvimento (segundo Ahlstrom \& Moser, 1976), que consiste na comparação morfológica de indivíduos menores a partir da forma mais desenvolvida. Posteriormente, estas foram classificadas em quatro estágios (larval vitelino, pré-flexão, flexão e pós-flexão) de acordo com o grau de flexão da notocorda e desenvolvimento da nadadeira caudal e seus elementos de suporte, segundo AhLSTROM \& BALL (1954), modificado por NAKATANI et al. (2001). A descrição de cada estágio foi baseada no grau de desenvolvimento e na ocorrência dos principais eventos morfológicos, sendo ilustrados, com auxílio de câmara clara, os indivíduos que melhor representassem estas características.

Para a caracterização do desenvolvimento inicial, foram tomadas, por meio de estereomicroscópio equipado com ocular micrométrica, as seguintes medidas corporais expressas em milímetros (AHLSTROM et al., 1976): comprimento padrão (CP), comprimento do focinho (CF), diâmetro do olho (DOL), comprimento da maxila (CMX), altura da cabeça (AC), comprimento da cabeça (CC), altura do corpo (ACO) e distâncias prépeitoral (FNP), pré-pélvica (FNV), pré-dorsal (FND) e pré-anal (FNA). Para a caracterização merística foram contados, sempre que possível, o número de miômeros total, pré- e pós-anal e os raios das nadadeiras peitoral (P), pélvica (V), dorsal (D) e anal (A). Para análise das relações corporais, as variáveis morfométricas foram expressas como porcentagens do comprimento padrão e comprimento da cabeça ao longo do desenvolvimento. As relações corporais para a altura do corpo, comprimento da cabeça e diâmetro do olho foram estabelecidas utilizando-se os critérios sugeridos por LeIS \& TRNSKI (1989).

Modelos de regressão. Para examinar possíveis variações ontogênicas ao longo do desenvolvimento da espécie, as variáveis morfométricas (variáveis resposta) foram plotadas contra os comprimentos padrão e da cabeça (variáveis explanatórias) e suas relações descritas por diferentes modelos de regressão (KovÁč et al., 1999). Inicialmente, a hipótese de que o desenvolvimento das relações corporais é isométrico contínuo foi testada a partir de uma regressão linear simples. Conjuntamente à hipótese de isometria contínua, foram testadas as hipóteses alternativas de desenvolvimento gradualmente alométrico (regressão quadrátrica) ou isometria descontínua (regressão linear por partes, do tipo piecewise, o qual é marcado por pontos de quebra que refletem diferentes taxas de crescimento). A seleção do melhor modelo para cada variável morfométrica em relação ao tamanho do corpo e da cabeça foi testada através de testes F (SoKal \& RohlF, 1981). O nível de significância adotado para as análises foi $\mathrm{p}<0,05$.

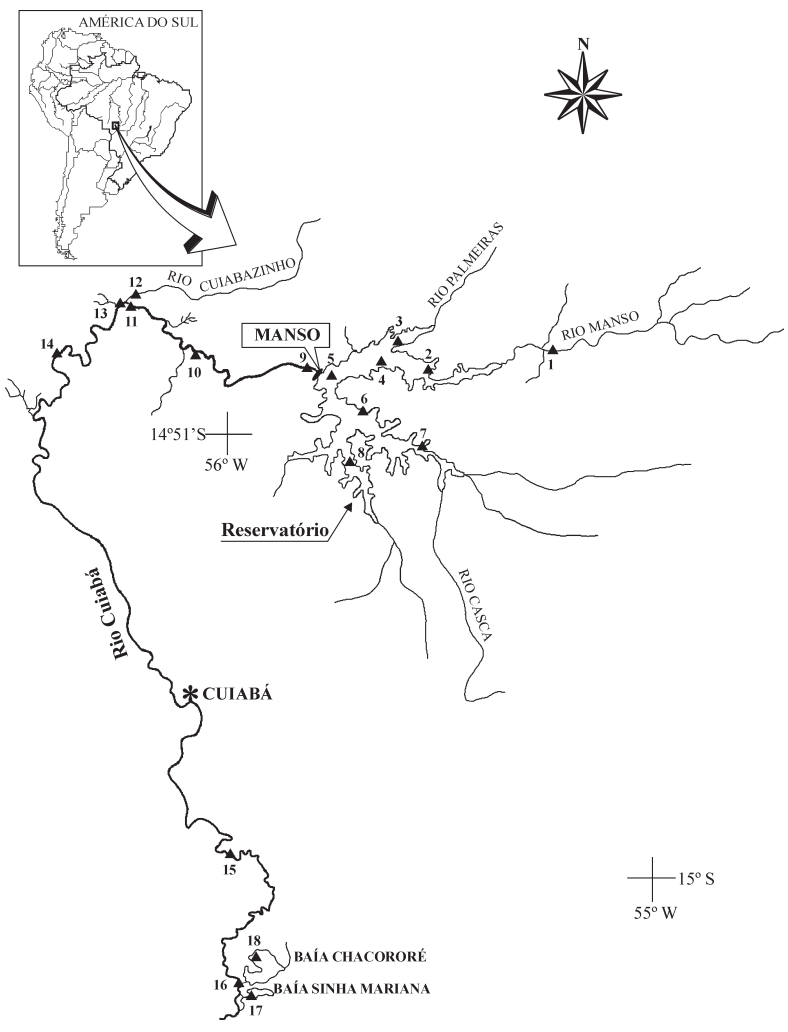

Fig. 1. Localização das estações de amostragem (triângulos) de larvas de Brycon hilarii (Valenciennes, 1850) nas bacias dos rios Cuiabá e Manso, Mato Grosso, Brasil, no período entre março de 2000 e março de 2004 . 


\section{RESULTADOS}

Estágios de desenvolvimento. Foram analisadas 53 larvas (18 no estágio larval vitelino, 2 em pré-flexão, 20 em flexão e 13 em pós-flexão). A descrição de cada estágio é apresentada a seguir e ilustrada nas figuras 2-8. Larval vitelino. Neste estágio o comprimento padrão varia de 3,25 a 5,49 mm. Inicialmente, as larvas são esbranquiçadas e quase não apresentam pigmentação pelo corpo (Figs 2, 3). Com cerca de 3,25 mm, alguns pigmentos puntiformes são observados ao longo do intestino. É característico, nos indivíduos mais desenvolvidos (a partir de $3,72 \mathrm{~mm}$ ), a presença de pigmentos puntiformes enfileirados desde a região terminal do intestino até o pedúnculo caudal. A partir de $4,20 \mathrm{~mm}$, observa-se pigmentação puntiforme no dentário e, com $5,49 \mathrm{~mm}$, alguns poucos pigmentos puntiformes são encontrados dispersos pela região lateral e ventral da cabeça. O intestino é longo, com sua parte terminal ultrapassando a metade do corpo. A abertura anal ocorre com 4,20 mm. A notocorda é visível por transparência e o olho, ainda despigmentado, é levemente elíptico. A câmara orbital é visível por transparência na região póstero-superior da cabeça (Figs 2, 3).

A cabeça apresenta-se encurvada para baixo e, a partir de 5,36 mm, alinha-se ao eixo longitudinal do corpo, porém, com a abertura bucal ainda permanecendo inferior a este eixo. A boca é ampla em relação ao tamanho do olho e da cabeça e já se encontra aberta. No final do estágio, por volta de $5,49 \mathrm{~mm}$, o olho apresentase levemente pigmentado e predominantemente esférico. O saco vitelínico é inicialmente alongado e parcialmente absorvido no final do estágio. Em sua região superior, é possível observar o início do desenvolvimento do botão da nadadeira peitoral (ainda sem raios) com cerca de 4,20 mm. O opérculo encontra-se em início de formação e os arcos branquiais estão presentes com $4,20 \mathrm{~mm}$ apresentando os filamentos branquiais diferenciados a partir de 4,90 mm de CP (Figs 2, 3).

A bexiga natatória é invisível por transparência (não se encontrando inflada). As aberturas nasais são simples e circulares. O órgão adesivo encontra-se logo acima dos olhos na região dorsal e anterior da cabeça. A membrana embrionária é hialina e está por toda a região ventral e parte da região dorsal, estendendo-se desde a margem anterior do saco vitelínico (circundando a região caudal) até a porção mediana dorsal do corpo. As demais nadadeiras não se apresentam delineadas, exceto a nadadeira peitoral que na região superior do saco vitelínico é possível observar o início do desenvolvimento do botão (ainda sem raios) com cerca de 4,20 mm. Os miômeros variam de 41 a 50 (24 a 30 pré-anal e 16 a 24 pós-anal) (Tab. I).

Pré-flexão. O comprimento padrão varia, neste estágio, de 8,10 a $8,60 \mathrm{~mm}$. A pigmentação, de maneira geral, é ausente pelo corpo, exceto alguns poucos pigmentos dendríticos no dentário e puntiformes e dendríticos na região mediana do intestino (Fig. 4). O olho é esférico e encontra-se totalmente pigmentado. A câmara orbital não é mais visível. A boca é ampla e terminal e os dentes são bem desenvolvidos no final desse estágio. Os dentes começam a se formar no dentário e pré-maxilar apresentando-se em duas fileiras em cada osso com formato cônico. O saco vitelino encontra-se absorvido e, em seu lugar, observa-se o intestino longo e completamente desenvolvido, com estrias transversais bem visíveis. A bexiga natatória encontra-se inflada e visível sob transparência nas larvas mais desenvolvidas
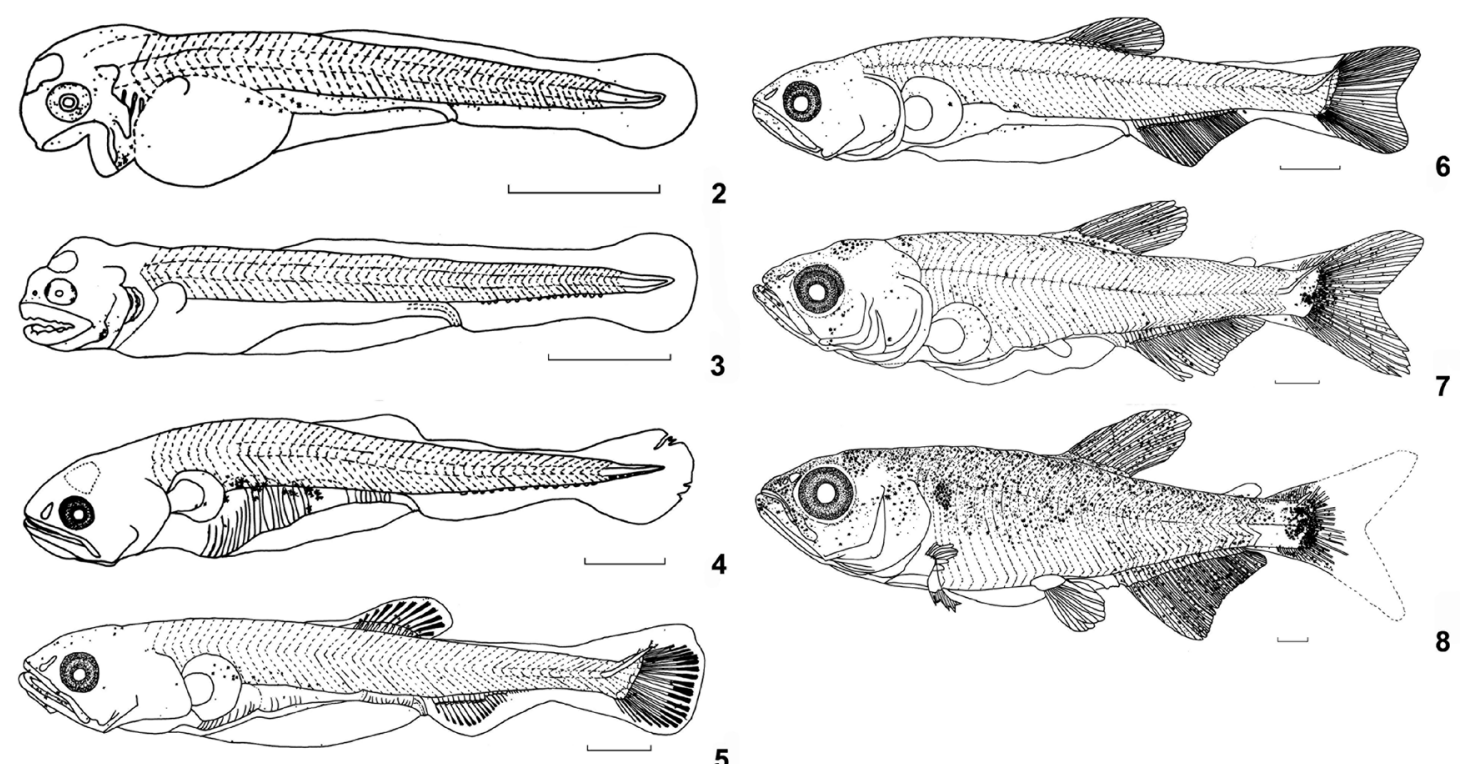

5

Figs 2-8. Desenvolvimento inicial de Brycon hilarii (Valenciennes, 1850) coletado nas bacias dos rios Cuiabá e Manso, Mato Grosso, Brasil, no período entre março de 2000 e março de 2004: 2, início de larval vitelino $(4,20 \mathrm{~mm} \mathrm{CP})$; 3 , final de larval vitelino $(5,49 \mathrm{~mm} \mathrm{CP}) ; 4$, pré-flexão $(8,60 \mathrm{~mm}) ; 5$, início de flexão $(10,04 \mathrm{~mm} \mathrm{CP}) ; 6$, final de flexão $(12,34 \mathrm{~mm} \mathrm{CP}) ; 7$, início de pós-flexão $(13,19 \mathrm{~mm} \mathrm{CP})$; 8, final de pós-flexão* $(17,84 \mathrm{~mm} \mathrm{CP})$. *A nadadeira caudal (tracejada na figura 8$)$ foi estimada devido à larva apresentar-se danificada. Barras $=1 \mathrm{~mm}$. 
Tab. I. Valores de comprimento (mm) mínimos (Min), máximos (Max), médias (x) e desvio padrão (DP) encontrados para as variáveis morfométricas e merísticas obtidas em larvas de Brycon hilarii (Valenciennes, 1850) (n, número de indivíduos analisados; LV, larval vitelino; PF, pré-flexão; FL, flexão; FP, pós-flexão), coletadas nas bacias dos rios Cuiabá e Manso, Mato Grosso, Brasil, no período entre março de 2000 e março de 2004 (CP, comprimento padrão; AC, altura da cabeça; CC, comprimento da cabeça; CF, comprimento do focinho; DOL, diâmetro do olho; CMX, comprimento da maxila; ACO, altura do corpo; FNP, distância focinho-nadadeira peitoral; FNV, distância focinho-nadadeira pélvica; FND, distância focinho-nadadeira dorsal; FNA, distância focinho-nadadeira anal; P, peitoral; V, pélvica; D, dorsal; A,anal). A ausência de medidas é devido ao grau de desenvolvimento incompleto das larvas.

\begin{tabular}{|c|c|c|c|c|c|c|c|c|}
\hline \multirow{3}{*}{$\begin{array}{c}\text { Variáveis } \\
(\mathrm{mm})\end{array}$} & \multicolumn{8}{|c|}{ Período Larval } \\
\hline & \multicolumn{2}{|c|}{$\mathrm{LV}(\mathrm{n}=18)$} & \multicolumn{2}{|c|}{$\mathrm{PF}(\mathrm{n}=2)$} & \multicolumn{2}{|c|}{$\mathrm{FL}(\mathrm{n}=20)$} & \multicolumn{2}{|c|}{$\mathrm{FP}(\mathrm{n}=13)$} \\
\hline & Min/Max & $\mathrm{x} \pm \mathrm{DP}$ & $\operatorname{Min} / \operatorname{Max}$ & $\mathrm{x} \pm \mathrm{DP}$ & Min/Max & $\mathrm{x} \pm \mathrm{DP}$ & $\operatorname{Min} / \operatorname{Max}$ & $\mathrm{x} \pm \mathrm{DP}$ \\
\hline $\mathrm{CP}$ & $3,25-5,49$ & $4,39-0,73$ & $8,10-8,60$ & $8,35-0,35$ & $8,00-10,94$ & $9,50-0,88$ & $11,09-26,00$ & $14,78-3,92$ \\
\hline $\mathrm{AC}$ & $0,59-1,65$ & $0,83-0,16$ & $1,36-1,54$ & $1,45-0,13$ & $1,31-2,10$ & $1,70-0,24$ & $2,10-6,00$ & $3,10-0,95$ \\
\hline $\mathrm{CC}$ & $0,54-1,07$ & $0,77-0,14$ & $1,95-2,03$ & $1,99-0,06$ & $1,79-3,40$ & $2,47-0,45$ & $3,40-9,15$ & $4,77-1,46$ \\
\hline $\mathrm{CF}$ & $0,07-0,44$ & $0,17-0,08$ & $0,5-0,5$ & $0,5-0,00$ & $0,48-1,05$ & $0,74-0,14$ & $0,75-2,40$ & $1,30-0,46$ \\
\hline DOL & $0,22-0,32$ & $0,26-0,03$ & $0,40-0,43$ & $0,42-0,02$ & $0,39-0,90$ & $0,57-0,13$ & $0,70-2,10$ & $1,13-0,36$ \\
\hline CMX & & & & & $0,78-1,43$ & $1,13-0,17$ & $0,95-2,20$ & $1,40-0,32$ \\
\hline $\mathrm{ACO}$ & $0,70-1,10$ & $0,91-0,13$ & $1,29-1,72$ & $1,51-0,30$ & $1,31-2,20$ & $1,78-0,26$ & $2,20-7,20$ & $3,35-1,21$ \\
\hline FNP & & & & & $1,83-3,60$ & $2,59-0,50$ & $3,50-8,70$ & $4,91-1,38$ \\
\hline FNV & & & & & & & $7,20-13,49$ & $10,19-2,24$ \\
\hline FND & & & & & $2,91-6,20$ & $5,27-0,82$ & $6,00-14,24$ & $8,12-2,12$ \\
\hline FNA & & & & & $3,74-7,80$ & $6,54-0,98$ & $6,40-17,24$ & $9,64-2,76$ \\
\hline \multicolumn{9}{|l|}{ Miômeros } \\
\hline Total & $41-50$ & $46,63-3,10$ & $47-47$ & $47,00-0,00$ & $44-49$ & $47-1,35$ & $43-49$ & $47-1,77$ \\
\hline Pré-anal & $24-30$ & $27,61-1,61$ & $25-26$ & $25,50-0,71$ & $23-27$ & $25-1,24$ & $24-27$ & $25-0,99$ \\
\hline Pós-anal & $16-24$ & $19,38-2,60$ & $21-22$ & $21,50-0,71$ & $20-23$ & $21-0,81$ & $19-23$ & $21-1,37$ \\
\hline \multicolumn{9}{|l|}{ Raios } \\
\hline $\mathrm{P}$ & & & & & & & 15 & \\
\hline $\mathrm{V}$ & & & & & & & 8 & \\
\hline $\mathrm{D}$ & & & & & $7-11$ & $10-1,04$ & $10-11$ & $11-0,33$ \\
\hline $\mathrm{A}$ & & & & & $6-23$ & $17-6,77$ & $24-30$ & $26-1,75$ \\
\hline
\end{tabular}

do estágio. As aberturas nasais são simples e alongadas e o órgão adesivo ainda encontra-se presente na região superior da cabeça acima do olho (Fig. 4).

$\mathrm{O}$ opérculo e a nadadeira peitoral são mais desenvolvidos que no estágio anterior. O opérculo cobre toda a câmara branquial estendendo-se até a base de inserção do botão da nadadeira peitoral. Com relação à membrana embrionária, esta mantém o mesmo padrão de distribuição do estágio anterior, porém, apresentase parcialmente absorvida, com delineamento das nadadeiras dorsal e anal. Na região ventral, pós abertura anal, observa-se a formação dos pterigióforos dos raios da nadadeira anal e, a partir de $8,10 \mathrm{~mm}$, são visíveis na porção terminal da notocorda, os ossos hipurais. A notocorda é visível por quase toda a extensão do corpo, porém é menos visível na região anterior onde os miômeros são mais desenvolvidos. O número total de miômeros é 47 ( 25 a 26 pré-anal e 21 a 22 pós-anal) (Tab. I).

Flexão. O comprimento padrão varia, neste estágio, de 8,00 a 10,94 $\mathrm{mm}$. A notocorda atinge a flexão total com 9,00 mm. Inicialmente, ela é visível por quase toda a sua extensão e depois é encoberta por tecido muscular $(9,30 \mathrm{~mm})$ permanecendo visível somente na região do pedúnculo caudal. No início deste estágio, os pigmentos dendríticos encontram-se distribuídos em torno da boca, no focinho e, juntamente com pigmentos puntiformes (predominantes), concentrados na região dorsal da cabeça (Figs 5, 6). Pigmentos dendríticos estão na região do dentário. São características, no final desse estágio, duas fileiras de pigmentos dendríticos ao longo da região mediana dorsal, desde a porção pós-cefálica (início do tronco) até o pedúnculo caudal. A partir de $10,49 \mathrm{~mm}$, foi observado concentrações de pigmentos (dendríticos) na parte interna do opérculo, nos arcos branquiais e nos filamentos brânquias. A pigmentação caudal (puntiforme) inicia-se próximo à porção final da notocorda $(8,20 \mathrm{~mm})$ e, sequencialmente, pigmentos dendríticos concentraram-se na região terminal do pedúnculo e na base da nadadeira caudal (Figs 5, 6).

A bexiga natatória é visível. A boca é ampla e terminal, estendendo-se obliquamente até a região inferior do olho, apresentando-se inclinada (oblíqua) em relação ao comprimento da cabeça. As aberturas nasais 
são alongadas e o olho, de maneira geral, é esférico e completamente pigmentado. Estrias transversais são visíveis ao longo do intestino e a abertura anal encontrase na linha transversal da porção terminal da nadadeira dorsal. A formação dos raios na nadadeira caudal é observada a partir de $8,20 \mathrm{~mm}$ e sua segmentação a partir de $9,30 \mathrm{~mm}$. Sequencialmente são formadas as nadadeiras dorsal e anal, com a base de sustentação dos raios dorsais visíveis a partir de $8,90 \mathrm{~mm}$. Os raios são flexíveis, não há formação de espinhos e nas nadadeiras dorsal e anal não se observa segmentação dos raios. No final do estágio, a membrana embrionária está quase completamente absorvida no dorso, sendo possível observar vestígios na região superior do pedúnculo caudal onde irá se formar a nadadeira adiposa. Na região ventral, essa membrana encontra-se moderadamente absorvida, mas ainda interliga a nadadeira anal com a caudal. Ela também está presente na região do intestino onde irá originar a nadadeira pélvica. O número total de miômeros varia de 44 a 49 (23 a 27 pré-anal e 20 a 23 pós-anal) (Tab. I).

Pós-flexão. Neste estágio, o comprimento padrão varia de 11,09 a $26,00 \mathrm{~mm}$. O padrão de pigmentação permanece semelhante ao estágio anterior, porém, com maior intensificação dos pigmentos principalmente na região dorsal, destacando-se a cabeça e o flanco acima da linha lateral (Figs 7, 8). Pigmentos puntiformes pequenos contornam as nadadeiras dorsal, anal e caudal, enquanto os dendríticos encontram-se espalhados entre os raios. A porção final do pedúnculo caudal é intensamente pigmentada com pigmentos dendríticos prolongando-se pelos raios medianos da nadadeira caudal. Três linhas de pigmentos dendríticos são evidentes, sendo uma delas desde a base da nadadeira anal até o pedúnculo caudal, outra na linha lateral e a terceira na região mediana dorsal do corpo. Uma mancha umeral transversal ovalada é observada a partir de 17,84 mm (Fig. 8).

A bexiga natatória é visível ao longo de todo o estágio de pós-flexão. $\mathrm{O}$ olho é esférico e a abertura bucal terminal, mas levemente voltada para cima. A dentição não variou ao longo dos estágios, tendo sempre duas fileiras de dentes de formato cônico nos ossos pré-maxilar e dentário. A abertura nasal simples é inicialmente alongada e estreita na região mediana da abertura (em forma de 8), característica esta pela qual origina, ao longo do desenvolvimento, abertura nasal dupla e circular $(17,84 \mathrm{~mm}$ aproximadamente), sendo o diâmetro da abertura superior maior que o da inferior. Com relação à membrana embrionária, esta se posiciona ventralmente ao longo do intestino até 19,49 $\mathrm{mm}$, enquanto que na região dorsal ela encontra-se totalmente absorvida. A formação da nadadeira pélvica tem início com 11,09 mm. A segmentação dos raios das nadadeiras peitoral e pélvica é observada com $26,0 \mathrm{~mm}$ e, nas nadadeiras caudal e dorsal, os raios formados apresentaram-se bifurcados. A posição das nadadeiras não se altera ao longo do desenvolvimento, exceto a peitoral, que migra para a região ventral à medida que o saco vitelínico é absorvido. A nadadeira dorsal encontra-se posicionada entre o plano vertical terminal da nadadeira pélvica (anterior ao ânus) e o plano vertical inicial da nadadeira anal (posterior ao ânus). A nadadeira caudal é do tipo homocerca furcada, ou seja, dividida em dois lobos simétricos (um superior e outro inferior). O número total de raios das nadadeiras no final deste estágio é: P. 15 , V. 8, D. 11, A. 30. O número de miômeros varia de 43 a 49 ( 24 a 27 pré-anal e 19 a 23 pós-anal) (Tab. I).

Relações corporais. Ao longo do desenvolvimento ontogênico, a variável comprimento da cabeça apresentou um aumento em relação ao comprimento padrão, variando

Tab. II. Valores das relações corporais (\%) mínimos (Min), máximos (Max), médias (x) e desvio padrão (DP) encontrados para as variáveis morfométricas e merísticas obtidas em larvas de Brycon hilarii (Valenciennes, 1850) (n, número de indivíduos analisados; LV, larval vitelino; PF, pré-flexão; FL, flexão; FP, pós-flexão), coletadas nas bacias dos rios Cuiabá e Manso, Mato Grosso, Brasil, no período entre março de 2000 e março de 2004 (CP, comprimento padrão; $\mathrm{AC}$, altura da cabeça; $\mathrm{CC}$, comprimento da cabeça; $\mathrm{CF}$, comprimento do focinho; $\mathrm{DOL}$, diâmetro do olho; $\mathrm{CMX}$, comprimento da maxila; ACO, altura do corpo; FNP, distância focinho-nadadeira peitoral; FNV, distância focinho-nadadeira pélvica; FND, distância focinho-nadadeira dorsal; FNA, distância focinho-nadadeira anal). A ausência de medidas é devido ao grau de desenvolvimento incompleto das larvas.

\begin{tabular}{|c|c|c|c|c|c|c|c|c|}
\hline \multirow{3}{*}{$\begin{array}{c}\text { Relações } \\
\text { corporais } \\
(\%)\end{array}$} & \multicolumn{8}{|c|}{ Período Larval } \\
\hline & \multicolumn{2}{|c|}{$\operatorname{LV}(\mathrm{n}=18)$} & \multicolumn{2}{|c|}{$\mathrm{PF}(\mathrm{n}=2)$} & \multicolumn{2}{|c|}{$\mathrm{FL}(\mathrm{n}=20)$} & \multicolumn{2}{|c|}{$\mathrm{FP}(\mathrm{n}=13)$} \\
\hline & Min/Max & $\mathrm{x} \pm \mathrm{DP}$ & $\operatorname{Min} / \operatorname{Max}$ & $\mathrm{x} \pm \mathrm{DP}$ & Min/Max & $\mathrm{x} \pm \mathrm{DP}$ & $\operatorname{Min} / \operatorname{Max}$ & $\mathrm{x} \pm \mathrm{DP}$ \\
\hline $\mathrm{AC} / \mathrm{CC}$ & $91,03-123,53$ & $108,70-10,27$ & $69,74-75,86$ & $72,80-4,33$ & $59,93-90,91$ & $69,62-8,81$ & $58,33-73,81$ & $65,20-3,98$ \\
\hline $\mathrm{CF} / \mathrm{CC}$ & $11,11-41,12$ & $21,97-6,97$ & $24,63-25,64$ & $25,14-0,71$ & $23,33-44,12$ & $30,06-5,30$ & $20,00-33,33$ & $26,91-3,12$ \\
\hline $\mathrm{DOL} / \mathrm{CC}$ & $27,27-42,86$ & $34,34-4,15$ & $19,70-22,05$ & $20,88-1,66$ & $19,80-31,82$ & $23,01-3,39$ & $20,59-26,83$ & $23,59-1,49$ \\
\hline $\mathrm{CMX} / \mathrm{CC}$ & & & & & $39,67-57,65$ & $45,70-4,12$ & $12,35-37,73$ & $31,96-7,31$ \\
\hline $\mathrm{CC} / \mathrm{CP}$ & $14,36-20,73$ & $17,52-1,43$ & $23,60-24,07$ & $23,84-0,33$ & $20,97-31,87$ & $25,89-2,89$ & $27,54-35,19$ & $32,06-2,01$ \\
\hline $\mathrm{ACO} / \mathrm{CP}$ & $16,14-25,91$ & $20,88-2,28$ & $15,93-20,00$ & $17,96-2,88$ & $15,98-20,92$ & $18,68-1,47$ & $19,53-27,69$ & $22,75-2,24$ \\
\hline $\mathrm{FNP} / \mathrm{CP}$ & & & & & $22,44-34,86$ & $26,87-3,35$ & $28,46-35,69$ & $33,17-1,92$ \\
\hline $\mathrm{FNV} / \mathrm{CP}$ & & & & & & & $51,10-54,65$ & $52,84-1,39$ \\
\hline $\mathrm{FND} / \mathrm{CP}$ & & & & & $31,29-57,77$ & $53,00-6,56$ & $48,65-57,33$ & $54,99-2,02$ \\
\hline FNA/CP & & & & & $40,22-71,30$ & $65,69-7,62$ & $44,48-68,67$ & $64,17-5,87$ \\
\hline
\end{tabular}


Tab III. Valores matemáticos e estatísticos das regressões linear (L), quadrática (Q) e linear por partes (S) para as variáveis morfométricas obtidas em relação ao comprimento da cabeça e padrão em larvas de Brycon hilarii (Valenciennes, 1850) coletadas nas bacias dos rios Cuiabá e Manso, Mato Grosso, Brasil, no período entre março de 2000 e março de 2004. $\mathrm{R}^{2}=$ coeficiente de determinação; MM, melhor modelo; PQ, ponto de quebra $(\mathrm{mm})$; a e b, parâmetros das regressões (a, coeficiente angular e b, coeficiente linear); $\mathrm{n}$, número de indivíduos analisados; CP, comprimento padrão; AC, altura da cabeça; CC, comprimento da cabeça; CF, comprimento do focinho; DOL, diâmetro do olho; CMX, comprimento da maxila; ACO, altura do corpo; FNP, distância focinho-nadadeira peitoral; FNV, distância focinho-nadadeira pélvica; FND, distância focinho-nadadeira dorsal; FNA, distância focinho-nadadeira anal. $(*) \mathrm{p}<0,05$. (**) o $\underline{\mathrm{n}}$ foi insuficiente para realizar o teste $\mathrm{F}$.

\begin{tabular}{|c|c|c|c|c|c|c|c|c|c|c|c|c|c|}
\hline \multirow{2}{*}{ Relação } & \multicolumn{3}{|c|}{$\mathrm{R}^{2}$} & \multicolumn{3}{|c|}{ teste $\mathrm{F}$} & \multirow[b]{2}{*}{ MM } & \multirow[b]{2}{*}{ PQ } & \multirow[b]{2}{*}{ a1 } & \multirow[b]{2}{*}{ b1 } & \multirow[b]{2}{*}{$\mathrm{a} 2$} & \multirow[b]{2}{*}{ b2 } & \multirow[b]{2}{*}{$\mathrm{n}$} \\
\hline & $L$ & $Q$ & $S$ & $Q / L$ & $S / Q$ & $S / L$ & & & & & & & \\
\hline $\mathrm{CF} / \mathrm{CC}$ & 0,94 & 0,94 & 0,95 & 1,25 & $8,83^{*}$ & $5,14^{*}$ & S & 0,65 & 0,30 & $-0,06$ & 0,24 & 0,15 & 53 \\
\hline $\mathrm{DOL} / \mathrm{CC}$ & 0,96 & 0,96 & 0,97 & $-6,95^{*}$ & $31,88^{*}$ & $10,28^{*}$ & S & 0,58 & 0,15 & 0,14 & 0,23 & 0,01 & 53 \\
\hline $\mathrm{AC} / \mathrm{CC}$ & 0,97 & 0,95 & 0,98 & $-23,39^{*}$ & $61,16^{*}$ & $4,22^{*}$ & $\mathrm{~S}$ & 1,73 & 0,49 & 0,44 & 0,63 & 0,16 & 52 \\
\hline $\mathrm{CMX} / \mathrm{CC}$ & 0,27 & 0,55 & 0,65 & $18,65^{*}$ & $7,56^{*}$ & $15,21^{*}$ & $\mathrm{~S}$ & 1,19 & 0,03 & 0,88 & 0,23 & 0,54 & 33 \\
\hline $\mathrm{CC} / \mathrm{CP}$ & 0,97 & 0,96 & 0,98 & $-14,87^{*}$ & $78,45^{*}$ & $20,03 *$ & $\mathrm{~S}$ & 2,38 & 0,29 & $-0,49$ & 0,38 & $-0,91$ & 53 \\
\hline $\mathrm{ACO} / \mathrm{CP}$ & 0,94 & 0,97 & 0,98 & $53,43 *$ & $23,83 *$ & $51,07^{*}$ & S & 1,84 & 0,16 & 0,22 & 0,32 & $-1,22$ & 53 \\
\hline $\mathrm{FNP} / \mathrm{CP}$ & 0,94 & 0,92 & 0,97 & $-7,45^{*}$ & $53,02 *$ & $16,33^{*}$ & $\mathrm{~S}$ & 3,36 & 0,40 & $-1,27$ & 0,33 & $-0,03$ & 34 \\
\hline $\mathrm{FNV/CP}$ & 0,99 & 0,99 & 1,00 & $-0,07$ & $* *$ & $* *$ & & & & & & & 3 \\
\hline $\mathrm{FND} / \mathrm{CP}$ & 0,94 & 0,94 & 0,95 & $-0,19$ & 3,71 & 1,75 & $\mathrm{~L}$ & & 0,56 & $-0,21$ & & & 27 \\
\hline FNA/CP & 0,94 & 0,94 & 0,95 & $-0,03$ & 2,12 & 1,04 & $\mathrm{~L}$ & & 0,67 & $-0,08$ & & & 27 \\
\hline
\end{tabular}

de pequena no estágio larval vitelino a grande em pósflexão (de 14,36 a 35,19\%, respectivamente) (Tab. II). O diâmetro do olho em relação ao comprimento da cabeça diminuiu nos primeiros estágios larvais (variando de 27,27 a $42,86 \%$ em larval vitelino e de 19,70 a $22,05 \%$ em pré-flexão). Entretanto, no final do desenvolvimento (pós-flexão) manteve-se de tamanho moderado (variou de 20,59 a 26,83\%). Já o corpo manteve, em relação ao comprimento padrão, altura moderada em larval vitelino $(16,14$ a $25,91 \%)$ e variou nos demais estágios de longo a moderado (mínimo de 15,93\% em pré-flexão e máximo de 27,69\% em pós-flexão) (Tab. II).

A variável altura da cabeça diminuiu até o estágio de flexão (108,70\% em larval vitelínico e 69,62\% em flexão), mantendo-se pouco variável de flexão a pósflexão $(65,20 \%)$ (Tab. II). O contrário foi observado para o comprimento do focinho em relação ao comprimento da cabeça, que apresentou um aumento de larval vitelino até flexão $(21,97 \%$ e $30,06 \%$, respectivamente) e uma diminuição em pós-flexão $(26,91 \%)$. O comprimento da maxila diminuiu de flexão a pós-flexão $(45,70 \%$ a 31,96\%) e a distância pré-peitoral teve um aumento de flexão a pós-flexão, variando de 22,44 a 35,69\%. As distâncias prédorsal e pré-anal não apresentaram variações consideráveis de flexão à pós-flexão $(53,00 \%$ a $54,99 \%$ e $65,69 \%$ a $64,17 \%$, respectivamente) (Tab. II). Para a distância prépélvica não foi possível fazer tal análise, pois só foi viável medi-la no último estágio de desenvolvimento.

Com relação às análises do padrão de crescimento corporal, foi verificado ao longo do desenvolvimento que o comprimento do focinho, o diâmetro do olho, a altura da cabeça e o comprimento da maxila apresentaram desenvolvimento isométrico descontínuo (regressão linear por partes) em relação ao comprimento da cabeça, ou seja, ocorreu uma mudança abrupta de crescimento a partir de um determinado tamanho (ponto de quebra $=0,65 \mathrm{~mm}, 0,58 \mathrm{~mm}, 1,73 \mathrm{~mm}$ e 1,19 $\mathrm{mm}$, respectivamente) (Tab. III) que correspondeu aos tamanhos situados dentro do estágio de flexão (Tab. II). Após este ponto de quebra as variáveis diâmetro do olho, altura da cabeça e comprimento da maxila apresentaram incremento no crescimento $(\mathrm{a} 1=0,15$ $\mathrm{mm}, 0,49 \mathrm{~mm}, 0,03 \mathrm{~mm}$ e a2 $=0,23 \mathrm{~mm}, 0,63 \mathrm{~mm}, 0,23$ $\mathrm{mm}$, respectivamente), enquanto que o comprimento do focinho diminuiu o seu crescimento $(\mathrm{a} 1=0,30 \mathrm{~mm}$ e $\mathrm{a} 2=$ $0,24 \mathrm{~mm})$.

Para as variáveis que foram relacionadas ao comprimento padrão, foi verificado que o comprimento da cabeça, a altura do corpo e a distância pré-peitoral também apresentaram descontinuidade isométrica durante o desenvolvimento (regressão linear por partes; ponto de quebra $=2,38 \mathrm{~mm}, 1,84 \mathrm{~mm}$ e $3,36 \mathrm{~mm}$, respectivamente) (Tab. III). Com relação à velocidade de crescimento, o comprimento da cabeça e a altura do corpo apresentam um incremento a partir de flexão (a1 $=0,29 \mathrm{~mm}, 0,16 \mathrm{~mm}$ e a2 $=0,38 \mathrm{~mm}, 0,32 \mathrm{~mm}$, respectivamente), praticamente duplicando a velocidade de crescimento da altura do corpo, enquanto que a distância pré-peitoral apresentou redução $(\mathrm{a} 1=0,40$ $\mathrm{mm}$ e a $2=0,33 \mathrm{~mm}$ ). As distâncias pré-dorsal e préanal revelaram desenvolvimento isométrico contínuo (regressão linear), ou seja, tiveram um incremento constante ao longo do desenvolvimento.

\section{DISCUSSÃO}

No primeiro estágio de desenvolvimento após a eclosão (larval vitelino) várias espécies de peixes apresentam-se pouco desenvolvidas. Em Characiformes é comum, nessa fase, a escassez de pigmentação nos olhos, além da boca e intestino não funcionais (NAKATANI et al., 2001). O pouco desenvolvimento corporal das 
larvas nesse estágio está intimamente relacionado com a estratégia reprodutiva da espécie. Espécies com ovócitos pequenos, característico em espécies migradoras e sem cuidado parental (VAzzoler, 1996), tal como B. hilarii (Graça \& Pavanelli, 2007), geralmente apresentam curtos períodos de incubação e consequentemente pouco desenvolvimento corporal (BlaXter, 1988; FuIMAN, 2002). Em estudos com outras espécies de Brycon (Lopes et al., 1995; Andrade-Talmelli et al., 2001; ReynalteTATAJE et al., 2004; Arias, 2006; Correia et al., 2010; Gomes et al., 2011), observa-se períodos embrionários relativamente curtos se comparados a outras espécies de peixes tropicais (ANDRADe-TALmelli et al., 2001), parecendo esta ser uma característica comum ao gênero, caracterizando o pouco desenvolvimento corporal do grupo no início da vida.

A abertura do intestino e a pigmentação dos olhos, observadas no final do estágio larval vitelínico, e a abertura da boca e a formação dos dentes, observados no final do estágio pré-flexão, são eventos que ocorrem quase que simultaneamente. Segundo LASKER et al. (1970), a pigmentação dos olhos e a abertura da boca e do intestino estão relacionadas com o início da alimentação exógena da larva. Em larvas de B. orbignyanus, estudadas por Reynalte-Tataje et al. (2004) e Maciel et al. (2010), e de B. orthotaenia, abordadas por Gomes et al. (2011), o canibalismo (alimentação exógena) começou a ser observado a partir de aproximadamente $5,65 \mathrm{~mm}, 6,28 \mathrm{~mm}$ e 4,58 $\mathrm{mm}$ de $\mathrm{CP}$, respectivamente, tamanhos estes intermediários aos estágios larval vitelino e pré-flexão descritos no presente estudo para B. hilarii, comparativamente. A rápida absorção do vitelo, ainda nas larvas vitelínicas, e o intestino bem desenvolvido e olhos pigmentados, em pré-flexão, caracterizam $B$. hilarii como precoce à alimentação exógena, apresentando curto período de alimentação mista (BALON, 1984a) quando comparado à duração deste período em outras espécies com diferentes estratégias reprodutivas, como Parauchenipterus galeatus (Linnaeus, 1766) (SANCHES et al., 1999), Auchenipterus osteomystax (MirandaRibeiro, 1918) e Hoplias aff. malabaricus (Bloch, 1794) (BiAletZKi et al., 2001; 2008), onde todas apresentaram alimentação mista até o estágio de flexão.

Sob o ponto de vista ecológico, pode-se afirmar que espécies migradoras apresentam evolutivamente um rápido desenvolvimento corporal para sobreviverem sem o cuidado parental, uma vez que essa rápida passagem pela fase crítica de transição alimentar (endógena para a exógena) refletiria a adaptação ecológica da espécie em superar a vulnerabilidade das larvas às pressões ambientais bióticas (predação) e abióticas (variações físico-químicas). Concomitantemente sob a luz da conservação, o rápido desenvolvimento embrionário é um fator importante na questão produção/tempo que pode ser utilizado para o recrutamento de alevinos em ambientes super explorados pela pesca intensiva.

Em estudos sobre alimentação de peixes, o tamanho e a forma do intestino são geralmente relacionados ao hábito alimentar das espécies. A presença de intestino longo nas larvas de $B$. hilarii, estendendo-se até após a metade do corpo, condiz com o padrão de morfologia intestinal apresentado pelas espécies de hábito alimentar onívoro.

A mácula caudal e umeral presente em $B$. hilarii parece ser característica do gênero, pois padrão semelhante foi descrito em larvas de B. orbignyanus por NAKATANi et al. (2001) na bacia do alto rio Paraná (PR). Em outras populações de $B$. orbignyanus de diferentes bacias do Estado de Minas Gerais (Maciel et al., 2010; CorReIa et al., 2010), observou-se, em todos os estágios, um padrão de distribuição de pigmentos similar ao descrito para $B$. hilarii, havendo apenas pequenas diferenças em intensidade de pigmentação, principalmente na região do saco vitelínico. A distribuição da pigmentação pelo corpo da larva é importante por representar um caráter taxonômico bastante utilizado na identificação de espécies (Nascimento \& Araújo-Lima, 1993; Meijide \& Guerrero, 2000; Godinho et al., 2003). De acordo com Kendall et al. (1984), cada espécie tem um padrão distinto de pigmentação cujo tamanho e a localização dos melanóforos são determinados geneticamente. Em larvas de Salminus spp., por exemplo, não é observado a mancha umeral (NAKATANI et al., 2001), sendo esta uma característica importante para a distinção entre as larvas dos gêneros Salminus e Brycon, uma vez que o restante da morfologia é muito similar. Em larvas de $B$. orbignyanus estudadas por CORREIA et al. (2010), observa-se intensa pigmentação no saco vitelínico, diferindo nesse aspecto das larvas de $B$. hilarii. No entanto, vale ressaltar que as intensidade de pigmentação das larvas pode variar conforme o hábitat (marginal ou pelágico) onde elas vivem (SANChes et al., 2001; NAKATANi et al., 2004). Contudo, apesar dessas variações de pigmentação, a presença, a forma e a localização de manchas de pigmentos são características genéticas das espécies e são potencialmente úteis na diferenciação taxonômica.

Nos primeiros estágios de vida, as larvas não possuem eficiente mobilidade natatória e ficam sujeitas à força da correnteza da água. Sob essas condições, o órgão adesivo observado até o estágio de pré-flexão tornase funcional quando essas larvas, logo após a eclosão, começam a derivar rio abaixo, funcionando como um importante órgão para a dispersão das larvas por aderilas na superfície por meio da tensão superficial da água ou até mesmo em outras estruturas flutuantes (SANTOS \& Godinho, 2002). Esse órgão também é encontrado em larvas de B. orthotaenia, Salminus brasiliensis (Cuvier, 1817), Astronotus ocellatus (Cuvier, 1829), Salminus hilarii Valenciennes, 1850 e Astyanax altiparanae Garutti \& Britski, 2000 (NAKATANi et al., 2001).

Com relação à sequência de formação das nadadeiras, o padrão de desenvolvimento é semelhante ao observado em larvas de outros Characiformes (OldDANI, 1977, 1983; Araújo-Lima, 1985; Nascimento \& Araújo-Lima, 1993; SAnChes et al., 2001; Galuch et al., 2003; BialetzKi et al., 
1998; 2008), iniciando-se pela caudal seguido pela dorsal, anal, pélvicas e peitorais. O número de raios das nadadeiras dorsal, peitoral, anal e pélvica em pós-flexão coincidiu com aqueles apresentados pelos adultos em Graça \& PAVAnelli (2007) e BRITSKi et al. (2007).

As relações corporais apresentaram importantes mudanças durante o desenvolvimento ontogênico sob o ponto de vista adaptativo da espécie. A teoria saltatória da ontogenia (BALON, 1984b) descreve que o desenvolvimento larval constitui uma sequência longa de etapas estáveis que são interrompidas por uma rápida alteração no seu desenvolvimento, caracterizando uma fase de metamorfose larval. No entanto, essa fase de metamorfose só é significativa se puder ser associada a algum fator de alteração morfológica, fisiológica, ecológica ou comportamental da espécie (KovÁc et al., 1999). Para todas as variáveis analisadas (exceto as distâncias pré-dorsal e pré-anal), as grandes mudanças morfológicas (metamorfose) ocorreram no estágio de flexão e na transição para pós-flexão. Essas transformações, como por exemplo, a flexão da notocorda, a formação dos raios das nadadeiras e o maior desenvolvimento muscular (diminuição da transparência dos miômeros), juntas, propiciam um corpo mais apto para maior atividade natatória e busca de alimento.

Sob o sentido da teoria de BALON (1984b) e da análise de regressão de KovÀc et al. (1999) observase que o aumento da velocidade de crescimento do diâmetro do olho e da altura da cabeça estão associados, respectivamente, à mudança do hábito alimentar do peixe e ao desenvolvimento da massa cerebral e ósseo da região bucal (maxila e mandíbula amplas e obliquamente direcionadas à parte inferior da cabeça). Segundo Poullly et al. (2003), a variação do diâmetro do olho proporciona maior acuidade visual na procura do alimento e consequente aumento de itens alimentares na dieta. Em relação ao tamanho da cabeça, o desenvolvimento da parte cerebral (sistema nervoso central) leva à diversificação das habilidades motoras e sensoriais, podendo ocasionar mudanças de hábitos como o comportamento agressivo (caça) (SAKAmURA \& Тзикамото, 2002), possível preferência de hábitat (desenvolvimento da natação) e alimentação da espécie. Com relação ao comprimento da cabeça, o aumento apresentado ao longo do desenvolvimento em $B$. hilarii pode estar refletindo o desenvolvimento das brânquias e a expansão da câmara opercular. Fuiman et al. (1983) relacionaram o desenvolvimento dessa variável com o aumento da eficiência da respiração branquial, substituindo gradativamente a respiração cutânea das larvas. A variação do comprimento do focinho estaria vinculada a mudanças de hábitos alimentares que podem ocorrer durante o desenvolvimento larval, uma vez que, os ossos da boca (maxilar, pré-maxilar e mandíbula) ainda se encontram em formação e o tamanho do alimento ingerido pelas larvas pode variar concomitantemente com o tamanho da abertura bucal.
Este estudo enfatiza a importância de caracteres morfométricos e merísticos para o entendimento da biologia e caracterização das larvas de $B$. hilarii em ambientes naturais. $\mathrm{O}$ conhecimento das variações morfológicas durante o desenvolvimento ontogênico pode indicar adaptações ecológicas referentes a mudanças de hábitat, alimentação e comportamento das larvas de peixes. No caso de $B$. hilarii, as maiores modificações morfológicas (metamorfose) foram encontradas no estágio de flexão indicando que, possivelmente neste período, grandes mudanças comportamentais estejam ocorrendo. A morfologia, bem como a morfometria, são importantes ferramentas para auxiliar na diferenciação desta espécie de outras congêneres ou simpátricas. Entretanto, em alguns casos a níveis específicos, são necessários estudos mais aprofundados - como, por exemplo, o método de redes de treliça (STRAuss \& BoоKstein, 1982) -, que comparem o desenvolvimento inicial destas espécies de forma mais precisa, a fim de auxiliar na identificação desses organismos.

Agradecimentos. Os autores agradecem ao Convênio UEM/ Nupélia/Furnas Centrais Elétricas pelo apoio financeiro e ao Núcleo de Pesquisas em Limnologia, Ictiologia e Aquicultura (Nupélia) pelo suporte logístico; ao Programa Institucional de Bolsas de Iniciação Científica (PIBIC/UEM) e aos amigos do laboratório de Ictioplâncton/ Nupélia/UEM pelo auxílio nas atividades de laboratório.

\section{REFERÊNCIAS BIBLIOGRÁFICAS}

Ahlstrom, E. H. \& Ball, O. P. 1954. Description of eggs and larvae of jack mackerel (Trachurus symmetricus) and distribution and abundance of larvae in 1950 and 1951. Fisheries Bulletin 56:209245.

Ahlstrom, E. H. \& Moser, H. G. 1976. Eggs and larvae of fishes and their role in systematic investigations and in fisheries. Revue des Travaux de L'institut des Peches Maritimes 40(3/4):379-398.

Ahlstrom, E. H.; Butler, J. L. \& Sumida, B. Y. 1976. Pelagic stromateoid fishes (Pisces, Perciformes) of the Eastern Pacific: kinds, distributions, and early life histories and observations of live of these from the northwest Atlantic. Bulletin of Marine Science 40(3):285-402.

Andrade-Talmelli, E. F.; Kavamoto, E. T.; Romagosa, E. \& Fenerich-Verani, N. 2001. Embryonic and larval development of the "piabanha", Brycon insignis, Steidachner, 1876 (Pisces, Characidae). Boletim do Instituto de Pesca 27(1):21-28.

Araúuo-Lima, C. A. R. M. 1985. Aspectos biológicos de peixes amazônicos. V. Desenvolvimento larval do jaraqui-escama grossa, Semaprochilodus insignis (Characiformes, Pisces) da Amazônia Central. Revista Brasileira de Biologia 45(4):423-431.

ArIAs, A. M. 2006. Desarrollo larval de Brycon guatemalensis (Regan, 1908) (Pisces: Characidae). Brenesia 66:31-36.

BAlON, E. K. 1984a. Patterns in the evolution of reproductive styles in fishes. In: Potтs, G. W. \& Wооттоn, R. J. eds. Fish reproduction: strategies and tactics. London, Academic Press. p.35-53.

$1984 b$. Reflections on some decisive events in the early life of fishes. Transactions of the American Fisheries Society 113:178-185.

Banack, S. A.; Horn, M. H. \& GawlickA, A. 2002. Disperser-vs. establishment-limited distribution of a riparian fig tree (Ficus insipida) in a Costa Rican tropical rain forest. Biotropica 34(2):232-243.

Bialetzki, A.; Sanches, P. V.; Baumgartner, G. \& Nakatani, K. 1998. Caracterização morfológica e distribuição temporal de larvas e juvenis de Apareiodon affinis (Steindachner, 1879) (Osteichthyes, Parodontidae) no alto do rio Paraná (PR). Revista Brasileira de Zoologia 15(4):1037-1047.

Bialetzki, A.; Baumgartner, G.; Sanches, P. V.; Galuch, A. V.; Luvisuto, M. A.; Nakatani, K.; Makrakis, M. C. \& Borges, M. E. E. 2001. Caracterização do desenvolvimento inicial de 
Auchenipterus osteomystax (Osteichthyes, Auchenipteridae) da bacia do rio Paraná, Brasil. Acta Scientiarum, Biological Sciences 23(2):377-382.

Bialetzki, A.; Nakatani, K.; Sanches, P. V.; Baumgartner, G.; Makrakis, M. C. \& TAGUTI, T. L. 2008. Desenvolvimento inicial de Hoplias aff. malabaricus (Bloch, 1794) (Osteichthyes, Erythrinidae) da planície alagável do alto rio Paraná, Brasil. Acta Scientiarum, Biological Sciences 30(2):141-149.

BlaXter, J. H. S. 1988. Pattern and variety in development. In: Hoar, W. S. \& Randall, D. J. eds. Fish Physiology. London, Academic Press. v.11, p.1-58.

Britski, H. A.; Sato, Y. \& Rosa, A. B. S. 1988. Manual de identificação de peixes da região de Três Marias: (com chaves de identificação para os peixes da bacia do São Francisco). Brasília, CODEVASF. $115 \mathrm{p}$.

Britski, H. A.; Silimon, K. Z. S. \& Lopes, B. S. 2007. Peixes do Pantanal: Manual de identificação. Brasília, EMBRAPA. 227p.

Ceccarelli, P. S. \& Senhorini, J. A. 1996. Brycon: Viabilização da produção de alevinos. Panorama da Aqüicultura 6(35):10-11

Correia, M. A.; Maciel, C. M. R. R.; Nascimento, L. S. \& Maciel JR., A. 2010. Ontogenia da pigmentação das larvas de três espécies de Characiformes, criadas em incubatório. Enciclopédia Biosfera 6(11):1-14.

Faustino, F.; NaKaghi, L. S. O. \& Neumann, E. 2010. Brycon gouldingi (Teleostei, Characidae): aspects of the embryonic development in a new fish species with aquaculture potential. Zygote 19(4):351-363.

Froese, R. \& Pauly, D. 2011. A Global Information System on Fishes. Disponível em: <http://www.fishbase.org/Summary/ speciesSummary.php?ID=52994\&genusname $=$ Brycon\&speciesn ame $=$ hilarii $>$. Acesso em: 30.09 .2011 .

Fuiman, L. A. 2002. Special considerations of fish eggs and larvae. In: Fuiman, L. A. \& Werner, R. G. eds. Fishery Science: the unique contributions of early life stages. Oxford, Blackwell Science. p.1-32.

Fuiman, L. A.; Conner, J. V.; Lathrop, B. F.; Buynak, G. L.; Shyder, D. E. \& Loos, J. J. 1983. State of the art of identification for cyprinid fish larvae from eastern North America. Transactions of the American Fisheries Society 112:319-332.

Galuch,A. V.; Suiberto, M. R.; NAKatani, K.; BialetZki,A. \& Baumgartner, G. 2003. Desenvolvimento inicial e distribuição temporal de larvas e juvenis de Bryconamericus stramineus Eigenmann, 1908 (Osteichthyes, Characidae) na planície alagável do alto rio Paraná, Brasil. Acta Scientiarum, Biological Sciences 25(2):335-343.

Godinho, H. P.; Santos, J. E. \& SAto, Y. 2003. Ontogênese larval de cinco espécies de peixes do São Francisco. In: Godinho, H. P. \& GodinHo, A. L. orgs. Águas, peixes e pescadores do São Francisco das Minas Gerais. Belo Horizonte, PUC Minas. p.133-148.

Gomes, R. Z.; Sato, Y.; Rizzo, E. \& Bazzoli, N. 2011. Early development of Brycon orthotaenia (Pisces: Characidae). Zygote 7:1-10.

GraçA, W. J. \& Pavanelli, C. S. 2007. Peixes da planície de inundação do alto rio Paraná e áreas adjacentes. Maringá, EDUEM. 241p.

Howes, G. 1982. Review of the genus Brycon (Teleostei-Characoidei). Bulletin of British Museum of Natural History (Zoology) 43(1):1-47.

Kendall, A. W.; Ahlstrom, E. H. \& Moser, H. G. 1984. Ontogeny and systematics of fishes. Lawrence, American Society Icthyologists and Herpetologists. p.11-12.

Kováč, V.; Copp, G. H. \& Francis, M. P. 1999. Morphometry of the stone loach, Barbatula barbatula: do mensural characters reflect the species life history thresholds? Environmental Biology of Fishes 56:105-115.

Lasker, R.; Feder, H. M.; Theilack, G. H. \& May, R. C. 1970. Feeding, growth and survival of Engraulis mordax larvae reared in the laboratory. Marine Biology 5(4):345-353.

Leis, J. M. \& TRnski, T. 1989. The larvae of Indo-Pacific shorefishes. Honolulu, University of Hawaii Press; Sydney, The Australian Museum. 371p.

Lima, F. C. T. DE. 2003. Subfamily Bryconinae (Characins, tetras). In: Reis, R. E.; Kullander, S. O. \& Ferraris, Jr. C. J. eds. Check list of freshwater fishes of South and Central America. Porto Alegre, EDIPUCRS. p.174-181.
Lopes, R. N. M.; Senhorini, J. A. \& Soares, M. C. F. 1995. Desenvolvimento embrionário e larval do matrinxã Brycon cephalus Günther, 1869 (Pisces, Characidae). Boletim Técnico CEPTA 8:25-39.

Maciel, C. M. R. R.; Lanna, E. A. T.; Donzele, A. M. JR. J. L.; Neves, C. A. \& Menin, E. 2010. Morphological and behavioral development of the piracanjuba larvae. Revista Brasileira de Zootecnia 39(5):961-970.

Meijide, F. J. \& GuerRero, G. A. 2000. Embryonic and larval development of a substrate-brooding cichlid Cichlasoma dimerus Heckel, 1940 under laboratory conditions. Journal of Zoology 252:481-493.

Nakatani, K.; Bialetzki, A.; Baumgartner, G.; Sanches, P. V. \& Makrakis, M. C. 2004. Temporal and spatial dynamics of fish eggs and larvae. In: Thomaz, S. M.; Agostinho, A. A. \& Hahn, N. S. eds. The upper Paraná river and its floodplain: physical aspects, ecology and conservation. Leiden, Backhuys Publishers. p.293-308

Nakatani, K.; Agostinho, A. A.; Baumgartner, G.; Bialetzki, A.; Sanches, P. V.; Makrakis, M. C. \& Pavanelli, C. S. 2001. Ovos e larvas de peixes de água doce: desenvolvimento e manual de identificação. Maringá, EDUEM. 378p.

Nascimento, F. L. \& Araújo-Lima, C. A. R. M. 1993. Descrição de larvas de Psectrogaster amazonica e Potamorhina altamazonica (Curimatidae, Pisces) da Amazonia Central. Acta Amazonica 23(4):457-472.

Oldani, N. O. 1977. Identificación y morfologia de larvas de Apareiodon affinis (Steindachner, 1879) (Pisces, Parodontidae). Physis 37(93):133-140.

. 1983. Identificación y morfologia de larvas, juveniles y adultos de Mylossoma paraguayensis Norman, 1939 (Pises, Characidae). Studies on Neotropical Fauna and Environment 48:89-100.

Poullly, M.; Lino, F.; Bretenoux, J. G. \& Rosales, C. 2003. Dietary-morphological relationships in a fish assemblage of the bolivian amazonian floodplain. Journal of Fish Biology 62:1137-1158.

Reynalte-Tataje, D; Zaniboni-Filho, E. \& Esouivel, J. R. 2004. Embryonic and larvae development of piracanjuba, Brycon orbignyanus Valenciennes, 1849 (Pisces, Characidae). Acta Scientiarum, Biological Sciences 26(1):67-71.

Reys, P.; Sabino, J. \& Galetti, M. 2009. Frugivory by the fish Brycon hilarii (Characidae) in western Brazil. Acta Oecologica 35(1):136-141.

Romagosa, E.; Narahara, M. Y. \& Fenerech-Verani, N. 2001. Stages of embryonic development of the "Matrinxã", Brycon cephalus (Pisces: Characidae). Boletim do Instituto de Pesca 27(1):27:32.

Sakamura, Y. \& Tsukamoto, K. 2002. Onset and development of aggressive behavior in the early life stage of Japanese flounder. Fisheries Science 68:854-861.

Sanches, P. V.; Nakatani, K. \& Bialetzki, A. 1999. Morphological description of developmental stages of Parauchenipterus galeatus (Linnaeus, 1766) (Siluriformes, Auchenipteridae) on the floodplain of the upper Paraná River. Revista Brasileira de Biologia 59(3):429-438.

Sanches, P. V.; Baumgartner, G.; Bialetzki, A.; Suiberto, M. R.; Gomes, F. D. C.; Nakatani, K. \& Barbosa, N. D. C. 2001. Caracterização do desenvolvimento inicial de Leporinus friderici (Osteichthyes, Anostomidae) da bacia do rio Paraná, Brasil. Acta Scientiarum, Biological Sciences 23(2):383-389.

SAnTOS, J. E. \& Godinho, H. P. 2002. Ontogenetic events and swimming behavior of larvae of the characid fish Salminus brasiliensis (Cuvier) (Characiformes, Characidae) under laboratory conditions. Revista Brasileira de Zoologia 19:163-171.

SOKAL, R. R. \& RohlF, F. J. 1981. Biometry. The principles and practice of statistics in biological research. New York, W. H. Freenan and Company. 859p.

StRauss, R. E. \& BoOKSTEIN, F. L. 1982. The truss: body form reconstructions in morphometrics. Systematic Zoology 3(2):113-135.

VAzzoler, A. E. A. DE M. 1996. Biologia da reprodução de peixes teleósteos: teoria e prática. Maringá, EDUEM; São Paulo, SBI. 169 p. 